\title{
Checklist dos Trichoptera (Insecta) do Estado de São Paulo, Brasil
}

\author{
Adolfo Ricardo Calor ${ }^{1,2}$ \\ ${ }^{1}$ Laboratório de Entomologia Aquática, Departamento de Zoologia, Instituto de Biologia, \\ Universidade Federal da Bahia - UFBA, Campus Universitário de Ondina, Rua Barão de Geremoabo, s/n, \\ Ondina, CEP 40170-115, Salvador, BA, Brasil \\ ${ }^{2}$ Autor para correspondência: Adolfo Ricardo Calor, e-mail: acalor@gmail.com
}

CALOR, A.R. Checklist of Trichoptera (Insecta) from São Paulo State, Brazil. Biota Neotrop. 11(1a): http:// www.biotaneotropica.org.br/v11n1a/en/abstract?inventory+bn0311101a2011.

\begin{abstract}
Trichoptera are the major order among the aquatic insects and constitute a large proportion of benthic macroinvertebrate community. There are about 13,000 described species of caddisflies in the world, 2,500 in Neotropics and 479 species records to Brazil. The Brazilian caddisflies fauna included members of 16 families (Anomalopsychidae, Atriplectididae, Calamoceratidae, Ecnomidae, Glossosomatidae, Helicopsychidae, Hydrobiosidae, Hydropsychidae, Hydroptilidae, Leptoceridae, Limnephilidae, Odontoceridae, Philopotamidae, Polycentropodidae, Sericostomatidae and Xiphocentronidae). In São Paulo State, there are 126 registered species in 15 families, without records only to Limnephilidae.

Keywords: Trichoptera, biodiversity of the State of São Paulo, BIOTA/FAPESP Program.
\end{abstract}

Number of species: in the world: 14,254 (Morse 2010), in Brazil: 479, estimated in São Paulo State: 300.

CALOR, A.R. Checklist dos Trichoptera (Insecta) do Estado de São Paulo, Brasil. Biota Neotrop. 11(1a): http://www.biotaneotropica.org.br/v11n1a/pt/abstract?inventory+bn0311101a2011.

Resumo: Os Trichoptera compreendem a maior ordem de insetos estritamente aquáticos e constituem a maior proporção da comunidade dos macroinvertebrados bentônicos. Há 13.000 espécies descritas no mundo, cerca de 2.500 espécies na Região Neotropical e 479 ocorrentes no Brasil. A fauna brasileira de Trichoptera inclui membros de 16 famílias (Anomalopsychidae, Atriplectididae, Calamoceratidae, Ecnomidae, Glossosomatidae, Helicopsychidae, Hydrobiosidae, Hydropsychidae, Hydroptilidae, Leptoceridae, Limnephilidae, Odontoceridae, Philopotamidae, Polycentropodidae, Sericostomatidae e Xiphocentronidae). No Estado de São Paulo, há 126 espécies catalogadas em 15 famílias, sem registros apenas para Limnephilidae.

Palavras-chave: Trichoptera, biodiversidade do Estado de São Paulo, Programa BIOTA/FAPESP.

Número de espécies: no mundo: 14.254 (Morse 2010), no Brasil: 479, estimadas no Estado de São Paulo: 300. 


\section{Introdução}

Trichoptera Kirby 1813 compreendem a maior ordem de insetos estritamente aquáticos (Neboiss 1991) e constituem a maior proporção da comunidade dos macroinvertebrados bentônicos, com uma fauna mundial em torno de 13.000 espécies descritas para os ecossistemas dulcícolas (Holzenthal et al. 2007a, 2007b), além de algumas espécies marinhas da família Chathamiidae, encontradas na Nova Zelândia e Austrália (Neboiss 1991).

Na filogenia de Insecta, o posicionamento de Trichoptera tem alta estabilidade como grupo-irmão de Lepidoptera, desde os trabalhos de Tillyard (1935) e Ross (1967), passando por análises filogenéticas como Hennig (1969, 1981), Kristensen (1991), Wheeler et al. (2001) e Kjer (2004), entre tantos outros (para revisão veja Morse 1997). O clado Amphiesmenoptera (Trichoptera + Lepidoptera) tem mais de 20 sinapomorfias ( 21 com dados morfológicos apenas em Kristensen 1984) e a adição de novos dados vem aumentando a lista de homologias. Um caráter bastante notável na cladogênese de Amphiesmenoptera é a permeabilidade da parede da casa da pupa. A parede semipermeável é uma sinapomorfia de Trichoptera, que, provavelmente, capacitou os ancestrais da ordem a invadirem o ambiente aquático, fazendo desta a primeira, entre os Holometabola, a apresentar estágio pupal aquático.

Em relação aos sistemas classificatórios, os tricópteros são tradicionalmente organizados em quatro subordens: Protomeropina (=Permotrichoptera, segundo Eskov \& Sukatcheva 1997), Annulipalpia, Spicipalpia e Integripalpia. Protomeropina é composta de táxons fósseis (Permiano) e de posicionamento bastante controverso, sendo, algumas vezes, considerados representantes de Amphiesmenoptera stem group outras de grupos mais distantes filogeneticamente (Morse 1997). Apesar de controvérsias tanto na composição das subordens, quanto na relação filogenética entre elas, pode-se verificar a estabilidade do clado Integripalpia quanto a sua composição, o que não acontece com Annulipalpia (Calor 2009). No caso de Spicipalpia, as dúvidas estão tanto na monofilia do grupo, quanto no posicionamento de seus membros na filogenia de Trichoptera, de maneira que o status de infraordem (sensu Weaver III 1984) ou subordem (sensu Wiggins \& Wichard 1989) torna-se completamente duvidoso. Assim, entre as três subordens recentes de Trichoptera, apenas Integripalpia (sustentado em Weaver III 1984, Wiggins \& Wichard 1989, Frania \& Wiggins 1997, Ivanov 1997, Kjer et al. 2001, 2002, Holzenthal et al. 2007a) e Annulipalpia são grupos monofiléticos e, portanto, devem ser considerados táxons válidos (Calor 2009).

Para a região Neotropical, foram descritas 2.196 espécies de tricópteros (Flint et al. 1999), sendo que apenas 479 com ocorrência registrada para o Brasil. Estima-se que haja pelo menos mais de 300 novas espécies a serem descritas, depositadas em museus no Brasil e, principalmente, no exterior. A previsão está baseada no material depositado nas coleções, além da simples comparação com outras áreas da região Neotropical (e.g., Costa Rica apresenta 463 espécies descritas com área de apenas aproximadamente $51000 \mathrm{Km}^{2}$ ).

Atualmente, as 479 espécies conhecidas para o Brasil representam 16 famílias (Anomalopsychidae, Atriplectididae, Calamoceratidae, Ecnomidae, Glossosomatidae, Helicopsychidae, Hydrobiosidae, Hydropsychidae, Hydroptilidae, Leptoceridae, Limnephilidae, Odontoceridae, Philopotamidae, Polycentropodidae, Sericostomatidae e Xiphocentronidae), sendo que apenas uma delas (Limnephilidae) não apresenta registro para o Estado de São Paulo. A identificação dos táxons que ocorrem no país pode ser feita com auxílio das chaves de Angrisano \& Korob (2001) para América do Sul (larvas e adultos), Pes et al. (2005) para a região Amazônica (larvas), além de Calor (2007) e Froehlich et al. (no prelo) para as famílias ocorrentes no Brasil (larvas e adultos).

\section{Metodologia}

Esta checklist faz referência à literatura primária (artigos de descrição), fontes secundárias (checklists e artigos de distribuição geográfica) e aos dados originais oriundos das coletas do Projeto Temático BIOTA-FAPESP proc. 03/10517-9, sob coordenação do Dr. Claudio G. Froehlich (FFCLRP/USP) e da análise de material depositado em museus (especialmente no Museu de Zoologia da Universidade de São Paulo, Museu de Zoologia da Universidade Federal da Bahia e no Insect Museum of University of Minnesota).

Os casos de imprecisão quanto à localidade de coleta, em especial registros antigos com referência apenas ao país, não foram contabilizados para a somatória dos registros no Estado de São Paulo.

Trabalhos de cunho ecológico e/ou com identificação apenas até nível genérico (e.g., Pes et al. 2005, Spies \& Froehlich 2009) não foram considerados, visto que aqui é pretendido o conhecimento das espécies ocorrentes no Estado de São Paulo. O mesmo se aplica às possíveis espécies novas apenas citadas em teses, dissertações ou monografias.

Buscando compreender o conhecimento da tricopterofauna do Estado de São Paulo em relação ao existente para as demais unidades da federação, além do catálogo de Flint et al. (1999) e da checklist de Paprocki et al. (2004), também foram analisadas listas de distribuição publicadas (Dumas et al. 2009, 2010) ou apresentadas em congressos (Moretto et al. 2008). Artigos referenciados nestas publicações não foram relacionados nas referências bibliográficas deste texto.

Os tipos e os materiais determinados ou identificados encontramse depositados nas seguintes instituições ou acervos:

- AMNH - American Museum of Natural History, New York, New York, EUA.

- BMNH - The Natural History Museum, London, Inglaterra.

- CAS - California Academy of Sciences, San Francisco, California, EUA.

- CMP - Carnegie Museum, Pittsburgh, Pennsylvania, EUA.

- CNC - Canadian National Collection (Agriculture), Ottawa, Ontario, Canadá.

- CUI - Cornell University, Ithaca, New York, EUA.

- DEI - Deutsches Entomologisches Institut, Eberswalde, Alemanha.

- DZRJ - Coleção Entomológica Professor José Alfredo Pinheiro Dutra, Departamento de Zoologia, Universidade Federal do Rio de Janeiro, Rio de Janeiro, Brasil.

- DZUP - Coleção Pe. Jesus Santiago Moure, Departamento de Zoologia, Universidade Federal do Paraná, Curitiba, Brasil.

- IBUNAM - Instituto de Biología, Universidad Nacional Autonoma de México, Ciudad de México, México.

- IML - Instituto Miguel Lillo, Tucuman, Argentina.

- INHS - Illinois Natural History Survey, Urbana, Illinois, EUA.

- IRSNB - Institut Royal des Sciences Naturelles de Belgique, Brussels, Bélgica.

- IZAM - Instituto de Zoología Agrícola, Maracay, Venezuela.

- LACM - Los Angeles County Museum of Natural History, Los Angeles, California, EUA.

- MACN - Museo Argentino de Ciencias Naturales "Bernardino Rivadavia", Buenos Aires, Argentina.

- MCZ - Museum of Comparative Zoology, Harvard University, Cambridge, Massachusetts, EUA.

- MJP - Museo de Historia Natural "Javier Prado", Universidad Nacional de La Plata, La Plata, Argentina.

- MNHNP - Muséum National d'Histoire Naturelle, Paris, França.

- MNRJ - Museu Nacional, Universidade Federal do Rio de Janeiro, Rio de Janeiro, Brasil.

- MZBS - Museo de Zoologia, Barcelona, Espanha. 
- MZUSP - Museu de Zoologia, Universidade de São Paulo, São Paulo, Brasil.

- NMNH - National Museum of Natural History, Washington, DC, EUA.

- NMW - Naturhistorisches Museum Wien, Vienna, Áustria.

- PAN - Polish Academy of Sciences, Warsaw, Polônia.

- UFBA - Museu de Zoologia, Universidade Federal da Bahia, Salvador, Brasil.

- UMSP - University of Minnesota Insect Collection, Saint Paul, Minnesota, EUA.

- ZIUH - Zoologisches Institut, Universität Hamburg, Alemanha.

- ZSM - Zoologische Staatssamlung, München, Alemanha.

\section{Resultados e Discussão}

Ao início do Programa BIOTA-FAPESP, Froehlich (1999) listou 45 espécies conhecidas para o Estado de São Paulo. Paprocki et al. (2004) registraram 69 espécies com ocorrência no estado. Atualmente, há 126 registros de espécies para o Estado de São Paulo (Tabela 1), o que representa um aumento de aproximadamente $80 \%$. Este crescimento deve-se em parte aos projetos incluídos no Programa BIOTA-FAPESP (Processos 1998/05073-4 e 2003/10517-9), sob coordenação do Dr. Claudio G. Froehlich (FFCLRP/USP) e também ao projeto de levantamento da fauna de Trichoptera do sudeste brasileiro (National Science Foundation DEB 9971885), sob coordenação do Dr. Ralph W. Holzenthal (University of Minnesota, USA).

\section{Comentários sobre a lista, riqueza do estado comparada com outras regiões}

Como mencionado por Froehlich (no prelo), assim como o esperado para outras ordens de insetos aquáticos (excetuando-se os de interesse médico), o conhecimento taxonômico sobre os Trichoptera ainda é incipiente no Brasil. Entretanto, nos últimos anos há avanços significativos, principalmente nos estados de São Paulo, Minas Gerais, Rio de Janeiro e Amazonas, onde estão concentrados os principais grupos de pesquisa.

Paprocki et al. (2004) registraram 378 espécies com ocorrência em território nacional, havendo 69 registros para o Estado de São Paulo. No presente trabalho, os registros de espécies são contabilizados em 479 e 126 para o Brasil e São Paulo, respectivamente. Assim, em apenas seis anos, ocorreu um incremento de praticamente $80 \%$ dos registros de espécies no Estado de São Paulo e um aumento significativamente inferior (aproximadamente 25\%) quando considerado o território nacional como um todo. Outras unidades da federação que tiveram aumento significativo no mesmo período foram os estados do Amazonas (31\%), Minas Gerais (49,5\%) e Rio de Janeiro (66,5\%), com 143, 151 e 130 registros atuais, respectivamente.

Como pode ser visto na Tabela 2, apesar do avanço considerável nos últimos anos, o conhecimento da tricopterofauna está concentrado nos estados que sediam grupos de pesquisa e o acréscimo foi bem mais tímido nas demais unidades da federação. Com relação à região Nordeste do Brasil, por exemplo, há apenas dez registros de espécies

Tabela 1. Lista das espécies de Trichoptera do Estado de São Paulo.

Table 1. List of Trichoptera species from São Paulo State.

\begin{tabular}{|c|c|c|c|}
\hline $\begin{array}{c}\text { Família } \\
\text { (número de espécies) }\end{array}$ & Espécie & Descritor & $\begin{array}{c}\text { Espécimes em } \\
\text { Coleção Científica }\end{array}$ \\
\hline \multicolumn{4}{|l|}{ Anomalopsychidae (2) } \\
\hline & Contulma meloi & $\begin{array}{l}\text { Holzenthal \& Robertson } \\
2006\end{array}$ & 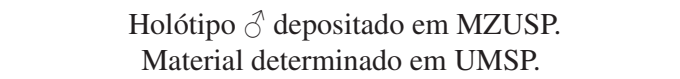 \\
\hline & Contulma tripui & $\begin{array}{l}\text { Holzenthal \& Robertson } \\
2006\end{array}$ & $\begin{array}{c}\text { Holótipo } \widehat{\jmath} \text { depositado em MZUSP. } \\
\text { Material determinado em MNRJ e UMSP. }\end{array}$ \\
\hline \multicolumn{4}{|l|}{ Calamoceratidae (8) } \\
\hline & Phylloicus abdominalis & (Ulmer 1905) & $\begin{array}{l}\text { Holótipo destruído. Neótipo depositado em MCZ. } \\
\text { Material determinado em BMNH, MCZ, MNRJ, } \\
\text { MZUSP, NMNH e UMSP. }\end{array}$ \\
\hline & Phylloicus angustior & Ulmer 1905 & $\begin{array}{l}\text { Holótipo } \widehat{\jmath} \text { depositado em NMW. Material determinado } \\
\text { em DEI, BMNH, MCZ, MZUSP, NMNH e UMSP. }\end{array}$ \\
\hline & Phylloicus bertioga & Prather 2003 & $\begin{array}{l}\text { Holótipo } \widehat{\jmath} \text { depositado em MZUSP. } \\
\text { Material determinado em NMNH. }\end{array}$ \\
\hline & Phylloicus bidigitatus & Prather 2003 & Holótipo $\widehat{o}$ depositado em NMW. \\
\hline & Phylloicus bromeliarum & Müller 1880 & $\begin{array}{l}\text { Lectótipo depositado em MCZ. Material determinado } \\
\text { em BMNH, DZRJ, MCZ, NMNH e ZIUH. }\end{array}$ \\
\hline & Phylloicus camargoi & $\begin{array}{l}\text { Quinteiro \& Calor } \\
2011\end{array}$ & $\begin{array}{c}\text { Holótipo } \widehat{\nwarrow} \text { depositado em MZUSP e parátipos em } \\
\text { MZUSP, UFBA e UMSP. }\end{array}$ \\
\hline & Phylloicus major & Müller 1880 & $\begin{array}{c}\text { Lectótipo depositado em MCZ. Material determinado } \\
\text { em BMNH, MCZ, NMNH, UFBA e UMSP. }\end{array}$ \\
\hline & Phylloicus quadridigitatus & Prather 2003 & Holótipo $\widehat{o}$ depositado em NMW. \\
\hline \multicolumn{4}{|l|}{ Ecnomidae (3) } \\
\hline & Austrotinodes bracteatus & Flint \& Denning 1989 & Holótipo $\widehat{o}$ depositado em MZUSP. \\
\hline & Austrotinodes longispinum & $\begin{array}{l}\text { Thomson \& Holzenthal } \\
2010\end{array}$ & 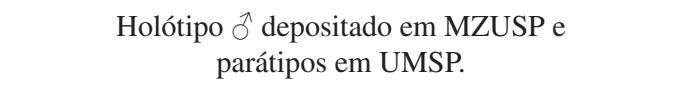 \\
\hline & Austrotinodes prolixus & $\begin{array}{l}\text { Flint \& Denning } \\
1989\end{array}$ & $\begin{array}{l}\text { Holótipo } \precsim \text { depositado em MZUSP. } \\
\text { Material determinado em DZRJ. }\end{array}$ \\
\hline
\end{tabular}


Calor, A.R.

Tabela 1. Continuação...

\begin{tabular}{|c|c|}
\hline $\begin{array}{c}\text { Família } \\
\text { (número de espécies) }\end{array}$ & Espécie \\
\hline \multicolumn{2}{|l|}{ Glossosomatidae (4) } \\
\hline & Canoptila williami \\
\hline & Mortoniella albolineata \\
\hline & Mortoniella teutona \\
\hline & Tolhuaca brasiliensis \\
\hline \multicolumn{2}{|l|}{ Helicopsychidae (3) } \\
\hline & $\begin{array}{c}\text { Helicopsyche (Cochliopsy- } \\
\text { che) clara }\end{array}$ \\
\hline & $\begin{array}{c}\text { Helicopsyche (Cochliopsy- } \\
\text { che) opalescens }\end{array}$ \\
\hline & $\begin{array}{c}\text { Helicopsyche (Feropsyche) } \\
\text { monda }\end{array}$ \\
\hline \multicolumn{2}{|l|}{ Hydrobiosidae (8) } \\
\hline & Atopsyche (Atopsaura) erigia \\
\hline & $\begin{array}{c}\text { Atopsyche (Atopsaura) } \\
\text { hatunpuna }\end{array}$ \\
\hline & $\begin{array}{c}\text { Atopsyche (Atopsaura) } \\
\text { hиапари }\end{array}$ \\
\hline & $\begin{array}{c}\text { Atopsyche (Atopsaura) } \\
\text { huarcu }\end{array}$ \\
\hline & $\begin{array}{c}\text { Atopsyche (Atopsaura) } \\
\text { plancki }\end{array}$ \\
\hline & $\begin{array}{c}\text { Atopsyche (Atopsaura) } \\
\text { sanctipauli }\end{array}$ \\
\hline & $\begin{array}{c}\text { Atopsyche (Atopsaura) } \\
\text { usingeri }\end{array}$ \\
\hline & Atopsyche (Atopsaura) zernyi \\
\hline
\end{tabular}

Hydropsychidae (33)

$$
\text { Blepharopus diaphanus }
$$

Centromacronema obscurum

Leptonema boraceia

Leptonema columbianum

Leptonema crassum

Leptonema pallidum

Leptonema serranum

Leptonema sparsum

Leptonema tridens
Robertson \& Holzenthal 2006

(Ulmer 1907)

(Mosely 1939)

Robertson \& Holzenthal 2005

(Ulmer 1905)

Flint 1972

Flint 1972

Ross 1947

Schmid 1989

Schmid 1989

Schmid 1989

Marlier 1964

Flint 1974

Denning \& Sikora 1968

Flint 1974

Kolenati 1859

(Ulmer 1905)

Flint, McAlpine \& Ross 1987

Ulmer 1905

Ulmer 1905

Guérin-Méneville 1843

Navás 1933

(Ulmer 1905)

Mosely 1933
Holótipo $\widehat{\jmath}$ depositado em INHS.

Holótipo $\widehat{\jmath}$ depositado em MZUSP. Material determinado em UFBA.

Holótipo $\widehat{\jmath}$ depositado em MZUSP. \}Material determinado em UFBA.

Holótipo $\widehat{\jmath}$ depositado em MZUSP. Material determinado em UFBA.

Holótipo $\widehat{\sigma}$ depositado em IRSNB.

Holótipo $\widehat{\jmath}$ depositado em NMW.

Holótipo ô depositado em CAS.

Holótipo đ̊ depositado em NMW.

Holótipo ô depositado em NMW. Material determinado em NMNH, UFBA e UMSP.

Holótipo ô depositado em BMNH.

Holótipo $\widehat{\jmath}$ depositado em MZUSP e parátipos em MZUSP e NMNH.

Lectótipo depositado em PAN.

Material determinado em BMNH, CMP, CNC, CUI, IZAM, MCZ, MZUSP e NMNH.

Holótipo ồ destruído. Material determinado em CNC, CUI, DEI, IBUNAM, IML, INHS, IZAM, MCZ, MNHNP, MZUSP E NMNH.

Lectótipo depositado em PAN. Material determinado em CNC, LACM, MACN, MZUSP e NMNH.

Lectótipo em ZIUH. Material determinado em NMW e ZIUH.

Holótipo $\widehat{\jmath}$ depositado em ZIUH. Material determinado em AMNH, CNC, INHS, IZAM, LACM, MCZ e NMNH.

Holótipo $\widehat{o}$ depositado em BMNH. Material determinado em MCZ, MNRJ, MZBS, MZUSP e NMNH.

Holótipo $\widehat{\jmath}$ depositado em MZUSP. 
Tabela 1. Continuação...

\begin{tabular}{|c|c|c|c|}
\hline $\begin{array}{c}\text { Família } \\
\text { (número de espécies) }\end{array}$ & Espécie & Descritor & $\begin{array}{c}\text { Espécimes em } \\
\text { Coleção Científica }\end{array}$ \\
\hline & Leptonema viridianum & Navás 1916 & $\begin{array}{l}\text { Holótipo o perdido. Material determinado em CAS, } \\
\text { CNC, INHS, LACM, MCZ e NMNH. }\end{array}$ \\
\hline & Macronema bicolor & Ulmer 1905 & $\begin{array}{l}\text { Lectótipo depositado em PAN. } \\
\text { Material determinado em DZRJ. }\end{array}$ \\
\hline & Macronema fulvum & Ulmer 1905 & Holótipo ổ depositado em ZIUH, destruído. \\
\hline & Macronema immaculatum & Mosely 1934 & $\begin{array}{l}\text { Holótipo } \widehat{\jmath} \text { depositado em BMNH. } \\
\text { Material determinado em UMSP. }\end{array}$ \\
\hline & Macrostemum diagramma & (McLachlan 1971) & Holótipo $\precsim$ depositado em BMNH. \\
\hline & Macrostemum hyalinum & (Pictet 1836) & Holótipo đ desconhecido. \\
\hline & Macrostemum maculatum & (Perty 1833) & $\begin{array}{l}\text { Lectótipo depositado em ZSM. } \\
\text { Material determinado em NMW. }\end{array}$ \\
\hline & Macrostemum par & (Navás 1930) & Holótipo $\widehat{\jmath}$ depositado em DEI. \\
\hline & Smicridea albosignata & Ulmer 1907 & Holótipo $\widehat{\jmath}$ depositado em ZIUH. \\
\hline & Smicridea bivittata & (Hagen 1861) & $\begin{array}{l}\text { Holótipo } \widehat{\jmath} \text { depositado em MCZ. } \\
\text { Material determinado em UMSP. }\end{array}$ \\
\hline & Smicridea coronata & Flint 1980 & $\begin{array}{l}\text { Holótipo } ð \text { depositado em NMNH. } \\
\text { Material determinado em MZUSP. }\end{array}$ \\
\hline & Smicridea dendifera & Flint 1983 & Holótipo $\widehat{ð}$ depositado em NMNH. \\
\hline & Smicridea froehlichi & $\begin{array}{l}\text { Almeida \& Flint } \\
2002\end{array}$ & $\begin{array}{c}\text { Holótipo } \delta \text { depositado em MZUSP e } \\
\text { parátipos em DZUP, MZUSP e NMNH. } \\
\text { Material determinado em DZUP, MZUSP e NMNH }\end{array}$ \\
\hline & Smicridea jundiai & $\begin{array}{l}\text { Almeida \& Flint } \\
2002\end{array}$ & $\begin{array}{c}\text { Holótipo } \precsim \text { depositado em MZUSP e parátipos em } \\
\text { DZUP e NMNH. Material determinado em DZUP, } \\
\text { DZRJ, MZUSP e NMNH. }\end{array}$ \\
\hline & Smicridea paranensis & Flint 1983 & $\begin{array}{l}\text { Holótipo } \widehat{ð} \text { depositado em NMNH. } \\
\text { Material determinado em UMSP. }\end{array}$ \\
\hline & Smicridea piraya & Flint 1983 & Holótipo $\precsim$ depositado em NMNH. \\
\hline & Smicridea radula & Flint 1974 & Holótipo $\widehat{\jmath}$ depositado em NMNH. \\
\hline & Smicridea ralphi & $\begin{array}{l}\text { Almeida \& Flint } \\
2002\end{array}$ & 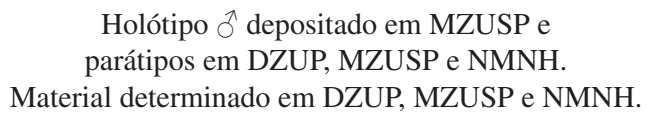 \\
\hline & Smicridea sattleri & Denning \& Sykora 1968 & Holótipo $\lesssim$ depositado em CAS. \\
\hline & Smicridea spinulosa & Flint 1972 & Holótipo $\widehat{\jmath}$ depositado em NMNH. \\
\hline & Smicridea unguiculata & Flint 1983 & Holótipo $\precsim$ depositado em NMNH. \\
\hline & Smicridea vermiculata & Flint 1983 & Holótipo $\widehat{\jmath}$ depositado em NMNH. \\
\hline & Synoestropsis pedicillata & Ulmer 1905 & Holótipo ô depositado em PAN. \\
\hline \multicolumn{4}{|l|}{ Hydroptilidae (8) } \\
\hline & Betrichia hamulifera & Flint 1983 & Holótipo $\lesssim$ depositado em NMNH. \\
\hline & Flintiella astilla & $\begin{array}{l}\text { Harris, Flint \& Holzenthal } \\
\qquad 2002\end{array}$ & $\begin{array}{l}\text { Holótipo } \widehat{\jmath} \text { depositado em NMNH e } \\
\text { parátipos em MZUSP e NMNH. }\end{array}$ \\
\hline & Flintiella boraceia & $\begin{array}{l}\text { Harris, Flint \& Holzenthal } \\
\qquad 2002\end{array}$ & Holótipo $\widehat{\jmath}$ depositado em MZUSP. \\
\hline & Hydroptila argentinica & Flint 1983 & Holótipo $\lesssim$ depositado em NMNH. \\
\hline & Oxyethira zilaba & (Mosely 1939) & Holótipo $\widehat{o}$ depositado em BMNH. \\
\hline & Rhyacopsyche bulbosa & $\begin{array}{l}\text { Wasmund \& Holzenthal } \\
\qquad 2007\end{array}$ & $\begin{array}{l}\text { Holótipo } \widehat{\jmath} \text { depositado em MZUSP e } \\
\text { parátipos em MZUSP e UMSP }\end{array}$ \\
\hline & Rhyacopsyche dikrosa & $\begin{array}{l}\text { Wasmund \& Holzenthal } \\
\qquad 2007\end{array}$ & $\begin{array}{l}\text { Holótipo } \widehat{\jmath} \text { depositado em MZUSP e } \\
\text { parátipos em MZUSP, NMNH e UMSP }\end{array}$ \\
\hline & Rhyacopsyche hagenii & Müller 1879 & Holótipo $\widehat{ð}$ perdido \\
\hline \multicolumn{4}{|l|}{ Leptoceridae (25) } \\
\hline & $\begin{array}{l}\text { Achoropsyche duodecim- } \\
\text { punctata }\end{array}$ & (Navás 1916) & $\begin{array}{c}\text { Holótipo } \widehat{ð} \text { perdido. Material determinado em } \\
\text { MZUSP, NMNH, UFBA e UMSP }\end{array}$ \\
\hline & Atanatolica brasiliana & (Brauer 1865) & $\begin{array}{l}\text { Holótipo } \delta \text { depositado em NMW. } \\
\text { Material determinado em MZUSP, NHW e UMSP }\end{array}$ \\
\hline
\end{tabular}


Calor, A.R.

Tabela 1. Continuação...

\begin{tabular}{|c|c|c|c|}
\hline $\begin{array}{c}\text { Família } \\
\text { (número de espécies) }\end{array}$ & Espécie & Descritor & $\begin{array}{c}\text { Espécimes em } \\
\text { Coleção Científica }\end{array}$ \\
\hline & Grumichella rostrata & Thienemann 1905 & $\begin{array}{c}\text { Holótipo } \widehat{\jmath} \text { desconhecido. Material determinado em } \\
\text { MZUSP, NMNH, UFBA e UMSP }\end{array}$ \\
\hline & Nectopsyche adusta & Flint 1983 & Holótipo $\widehat{\jmath}$ depositado em NMNH \\
\hline & Nectopsyche aureovittata & Flint 1983 & Holótipo $\widehat{\jmath}$ depositado em NMNH \\
\hline & Nectopsyche brunneofascia & Flint 1983 & Holótipo $\precsim$ depositado em NMNH \\
\hline & Nectopsyche flavofasciata & (Ulmer 1907) & $\begin{array}{l}\text { Lectótipo depositado em MCZ. } \\
\text { Material determinado em MCZ e MZBS }\end{array}$ \\
\hline & Nectopsyche fuscomaculata & Flint 1983 & Holótipo $\widehat{\jmath}$ depositado em NMNH \\
\hline & Nectopsyche gemmoides & Flint 1984 & Holótipo $\widehat{\jmath}$ depositado em NMNH \\
\hline & Nectopsyche muhni & (Navás 1916) & 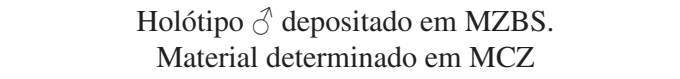 \\
\hline & Nectopsyche ortizi & Holzenthal 1995 & Holótipo $\widehat{o}$ depositado em NMNH \\
\hline & Nectopsyche pantosticta & Flint 1983 & Holótipo $\widehat{o}$ depositado em NMNH \\
\hline & Nectopsyche punctata & (Ulmer 1905) & 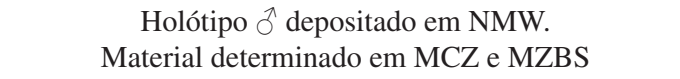 \\
\hline & Nectopsyche separata & (Banks 1920) & Holótipo đ̊ depositado em MCZ \\
\hline & Neoathripsodes anomalus & Holzenthal 1989 & $\begin{array}{l}\text { Holótipo } \widehat{\jmath} \text { depositado em MZUSP. } \\
\text { Material determinado em MZUSP, UFBA e UMSP }\end{array}$ \\
\hline & $\begin{array}{l}\text { Notalina (Neonotalina) } \\
\text { hamiltoni }\end{array}$ & Holzenthal 1986 & 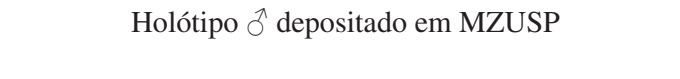 \\
\hline & $\begin{array}{c}\text { Notalina (Neonotalina) } \\
\text { morsei }\end{array}$ & Holzenthal 1986 & $\begin{array}{l}\text { Holótipo } \widehat{\jmath} \text { depositado em MZUSP. } \\
\text { Material determinado em MZUSP, UFBA e UMSP }\end{array}$ \\
\hline & $\begin{array}{l}\text { Notalina (Neonotalina) } \\
\text { paulista }\end{array}$ & Holzenthal 1986 & 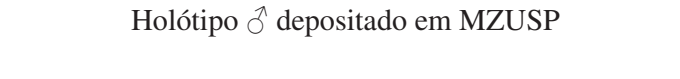 \\
\hline & Oecetis excisa & Ulmer 1907 & 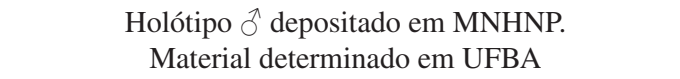 \\
\hline & Oecetis iguazu & Flint 1983 & Holótipo $\widehat{\jmath}$ depositado em NMNH \\
\hline & Oecetis inconspicua & (Walker 1852) & Holótipo $\widehat{o}$ depositado em BMNH \\
\hline & Oecetis punctipennis & (Ulmer 1905) & 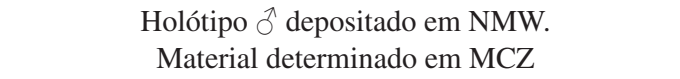 \\
\hline & Triplectides gracilis & (Burmeister 1839) & $\begin{array}{c}\text { Neótipo } \widehat{\text { }} \text { depositado em MZUSP. Material determinado } \\
\text { em MZUSP, NMNH, UFBA, UMSP e ZIUH }\end{array}$ \\
\hline & Triplectides misionensis & Holzenthal 1988 & $\begin{array}{l}\text { Holótipo ô depositado em NMNH. } \\
\text { Material determinado em DZRJ }\end{array}$ \\
\hline & Triplectides neotropicus & Holzenthal 1988 & Holótipo $\widehat{\jmath}$ depositado em NMNH \\
\hline \multicolumn{4}{|l|}{ Odontoceridae (2) } \\
\hline & Barypenthus concolor & Burmeister 1839 & $\begin{array}{c}\text { Lectótipo depositado em BNMH. Material determinado } \\
\text { em BMNH, DEI, MZUSP, UFBA, UMSP e ZIUH }\end{array}$ \\
\hline & Marilia albicornis & (Burmeister 1839) & Holótipo $\widehat{\partial}$ destruído \\
\hline \multicolumn{4}{|l|}{ Philopotamidae (21) } \\
\hline & Alterosa bocainae & Blahnik 2005 & $\begin{array}{l}\text { Holótipo } \overbrace{}^{\Uparrow} \text { depositado em MZUSP e } \\
\text { parátipos em MZUSP, NMNH e UMSP }\end{array}$ \\
\hline & Alterosa boraceiae & Blahnik 2005 & $\begin{array}{l}\text { Holótipo } \precsim \text { depositado em MZUSP e } \\
\text { parátipos em NMNH e UMSP }\end{array}$ \\
\hline & Alterosa escova & Blahnik 2005 & $\begin{array}{l}\text { Holótipo } \widehat{\jmath} \text { depositado em MZUSP e } \\
\text { parátipos em NMNH e UMSP }\end{array}$ \\
\hline & Alterosa falcata & Blahnik 2005 & $\begin{array}{l}\text { Holótipo } \widehat{\AA} \text { depositado em MZUSP e } \\
\text { parátipos em MZUSP, NMNH e UMSP }\end{array}$ \\
\hline & Alterosa intervalis & Blahnik 2005 & $\begin{array}{l}\text { Holótipo }{ }^{\Uparrow} \text { depositado em MZUSP e } \\
\text { parátipos em MZUSP e UMSP }\end{array}$ \\
\hline & Alterosa jordaensis & Blahnik 2005 & Holótipo $\widehat{\jmath}$ depositado em MZUSP \\
\hline & Alterosa sanctipauli & (Flint 1971) & Holótipo $\widehat{\jmath}$ depositado em NMNH \\
\hline & Alterosa truncata & Blahnik 2005 & $\begin{array}{l}\text { Holótipo } \widehat{\overbrace{}} \text { depositado em MZUSP e } \\
\text { parátipos em MZUSP, NMNH e UMSP }\end{array}$ \\
\hline
\end{tabular}


Tabela 1. Continuação...

\begin{tabular}{|c|c|c|c|}
\hline $\begin{array}{c}\text { Família } \\
\text { (número de espécies) }\end{array}$ & Espécie & Descritor & $\begin{array}{c}\text { Espécimes em } \\
\text { Coleção Científica }\end{array}$ \\
\hline & $\begin{array}{l}\text { Chimarra }(\text { Chimarra }) \\
\text { adamsae }\end{array}$ & Blahnik 1998 & $\begin{array}{l}\text { Holótipo } \widehat{o} \text { depositado em MJP e } \\
\text { parátipos em NMNH e UMSP }\end{array}$ \\
\hline & $\begin{array}{l}\text { Chimarra }(\text { Chimarrita }) \\
\text { camella }\end{array}$ & Blahnik 1997 & $\begin{array}{l}\text { Holótipo } \widehat{ð} \text { depositado em MZUSP e } \\
\text { parátipos em NMNH e UMSP }\end{array}$ \\
\hline & $\begin{array}{l}\text { Chimarra (Chimarrita) } \\
\text { camura }\end{array}$ & Blahnik 1997 & $\begin{array}{c}\text { Holótipo } \widehat{\partial} \text { depositado em MZUSP e } \\
\text { parátipos em NMNH }\end{array}$ \\
\hline & $\begin{array}{l}\text { Chimarra }(\text { Chimarrita }) \\
\text { kontilos }\end{array}$ & Blahnik 1997 & $\begin{array}{l}\text { Holótipo } \widehat{ð} \text { depositado em MZUSP e } \\
\text { parátipos em NMNH e UMSP }\end{array}$ \\
\hline & $\begin{array}{l}\text { Chimarra (Chimarrita) } \\
\text { majuscula }\end{array}$ & Blahnik 1997 & $\begin{array}{l}\text { Holótipo } \widehat{ð} \text { depositado em MZUSP e } \\
\text { parátipos em NMNH e UMSP }\end{array}$ \\
\hline & Chimarra (Curgia) beckeri & Flint 1998 & $\begin{array}{l}\text { Holótipo } \delta \text { depositado em MZUSP e parátipos em } \\
\text { MZUSP e NMNH. Material determinado em DZRJ }\end{array}$ \\
\hline & Chimarra (Curgia) boraceia & Flint 1998 & $\begin{array}{l}\text { Holótipo } \widehat{\overbrace{}} \text { depositado em MZUSP e } \\
\text { parátipos em MZUSP e NMNH }\end{array}$ \\
\hline & Chimarra (Curgia) froehlichi & Flint 1998 & $\begin{array}{l}\text { Holótipo ô depositado em MZUSP e } \\
\text { parátipos em MZUSP e NMNH }\end{array}$ \\
\hline & Chimarra (Curgia) hyoeides & Flint 1998 & $\begin{array}{l}\text { Holótipo ô depositado em NMNH. } \\
\text { Material determinado em MZUSP }\end{array}$ \\
\hline & Chimarra (Curgia) morio & (Burmeister 1839) & $\begin{array}{c}\text { Síntipo (provável) } \overbrace{}^{\Uparrow} \text { depositado em MCZ. } \\
\text { Material determinado em BMNH, CUI e MZUSP }\end{array}$ \\
\hline & Chimarra (Curgia) parana & Flint 1998 & $\begin{array}{l}\text { Holótipo } \widehat{\widehat{\jmath}} \text { depositado em NMNH. } \\
\text { Material determinado em MZUSP e NMNH }\end{array}$ \\
\hline & Chimarra (Curgia) teresae & Flint 1998 & 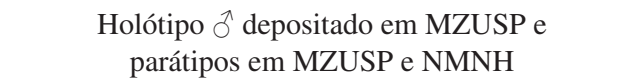 \\
\hline & Chimarra (Otarrha) odonta & Flint 2002 & $\begin{array}{l}\text { Holótipo } \widehat{\delta} \text { depositado em MZUSP e } \\
\text { parátipos em MZUSP, NMNH e UMSP }\end{array}$ \\
\hline \multicolumn{4}{|l|}{$\begin{array}{l}\text { Polycentropodidae } \\
\text { (6) }\end{array}$} \\
\hline & Cyrnellus mammillatus & Flint 1971. & $\begin{array}{l}\text { Holótipo ô depositado em NMNH. } \\
\text { Material determinado em DZRJ }\end{array}$ \\
\hline & Polyplectropus alatespinus & $\begin{array}{l}\text { Chamorro \& Holzenthal, } \\
\qquad 2010\end{array}$ & 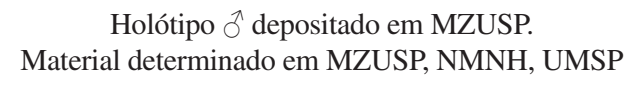 \\
\hline & Polyplectropus matatlanticus & $\begin{array}{l}\text { Chamorro \& Holzenthal, } \\
\qquad 2010\end{array}$ & 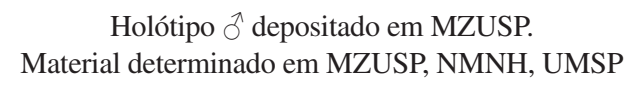 \\
\hline & Polyplectropus rodmani & $\begin{array}{l}\text { Chamorro \& Holzenthal, } \\
2010\end{array}$ & $\begin{array}{l}\text { Holótipo } \precsim \text { depositado em MZUSP. } \\
\text { Material determinado em MZUSP, NMNH, UMSP }\end{array}$ \\
\hline & Polyplectropus brasilensis & $\begin{array}{l}\text { Chamorro \& Holzenthal, } \\
2010\end{array}$ & $\begin{array}{l}\text { Holótipo } \widehat{\jmath} \text { depositado em MZUSP. } \\
\text { Material determinado em MZUSP, NMNH, UMSP }\end{array}$ \\
\hline & Polyplectropus tragularius & $\begin{array}{l}\text { Chamorro \& Holzenthal, } \\
2010\end{array}$ & 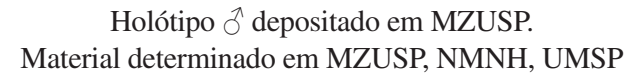 \\
\hline \multicolumn{4}{|l|}{ Sericostomatidae (1) } \\
\hline & Grumicha grumicha & (Vallot 1855) & $\begin{array}{l}\text { Lectótipo depositado em PAN. } \\
\text { Material determinado em MZUSP e UFBA }\end{array}$ \\
\hline \multicolumn{4}{|l|}{ Xiphocentronidae (2) } \\
\hline & Xiphocentron ilionea & Schmid 1982 & $\begin{array}{l}\text { Holótipo ô depositado em NMNH. } \\
\text { Material determinado em UMSP }\end{array}$ \\
\hline & Xiphocentron steffeni & (Marlier 1964) & Holótipo $\widehat{ð}$ depositado em IRSNB \\
\hline
\end{tabular}


Tabela 2. Registro atual das espécies de Trichoptera para os estados brasileiros em comparação com os dados apresentados em Paprocki et al. (2004).

Table 2. Number of Trichoptera species recorded per state in Brazil in comparison with Paprocki et al. (2004).

\begin{tabular}{|c|c|c|}
\hline Paprocki et al. (2004) & Número de espécies registradas & Estados \\
\hline 0 & 24 & $\mathrm{AC}$ \\
\hline 0 & 0 & AL \\
\hline 0 & 0 & AP \\
\hline 109 & 143 & $\mathrm{AM}$ \\
\hline 8 & 13 & BA \\
\hline 1 & 0 & $\mathrm{CE}$ \\
\hline 8 & 8 & DF \\
\hline 12 & 18 & ES \\
\hline 11 & 12 & GO \\
\hline 0 & 0 & MA \\
\hline 8 & 11 & MT \\
\hline 3 & 8 & MS \\
\hline 101 & 151 & MG \\
\hline 54 & 67 & $\mathrm{PA}$ \\
\hline 1 & 1 & PB \\
\hline 55 & 59 & PR \\
\hline 0 & 0 & $\mathrm{PE}$ \\
\hline 0 & 0 & PI \\
\hline 76 & 130 & RJ \\
\hline 0 & 0 & $\mathrm{RN}$ \\
\hline 4 & 4 & $\mathrm{RS}$ \\
\hline 14 & 17 & RO \\
\hline 17 & 18 & RR \\
\hline 97 & 113 & $\mathrm{SC}$ \\
\hline 69 & 126 & SP \\
\hline 0 & 0 & $\mathrm{SE}$ \\
\hline 0 & 0 & TO \\
\hline 378 & 479 & Brasil \\
\hline
\end{tabular}

de tricópteros (Paprocki et al. 2004), porém, com o desenvolvimento do projeto "Estudo taxonômico de Trichoptera Kirby, 1813: com ênfase na fauna da região Nordeste do Brasil" (CNPq) nos últimos dois anos, este valor se aproxima de uma centena (dados não incluídos na tabela).

\section{Principais avanços relacionados ao Programa BIOTA/FAPESP}

Como comentado anteriormente, foram registradas 126 espécies (69 sensu Paprocki et al. 2004) para o Estado de São Paulo, o que representa um aumento de aproximadamente $80 \%$ em seis anos, o maior entre todas as unidades da federação no mesmo período. Além dos trabalhos de taxonomia dos tricópteros (Calor \& Froehlich 2008), outros inferindo a filogenia destes insetos (Calor et al. 2006, Calor \& Holzenthal 2008, Calor 2009) também foram desenvolvidos no âmbito do programa.

Estudos de cunho ecológico realizados pela equipe dos projetos ligados ao Programa BIOTA-FAPESP, que incluíram os Trichoptera, representaram praticamente o início do desenvolvimento desta área de pesquisa no estado. Ainda dentro do referido programa, foi iniciada uma parceria com a Rede de Monitoramento de Qualidade das Águas Interiores da Companhia Ambiental do Estado de São Paulo (CETESB), com objetivo de implementar novos índices biológicos e integrar as informações sobre sedimento, testes de toxicidade e comunidades bentônicas no sistema de monitoramento da qualidade dos sistemas aquáticos do Estado de São Paulo.

\section{Principais grupos de pesquisa no país}

- Laboratório de Biologia Aquática, UNESP, campus de Assis, coordenado pelo Prof. Dr. Pitágoras da Conceição Bispo.

- Laboratório de Citotaxonomia e Insetos Aquáticos, Instituto Nacional de Pesquisas da Amazônia, coordenado pela Profa. Dra. Neusa Hamada e colaboração da Dra. Ana Maria de Oliveira Pes.

- Laboratório de Ecologia de Insetos, Departamento de Biologia Geral, Instituto de Ciências Biológicas, Universidade Federal de Goiás, coordenado pelo Prof. Dr. Adriano Sanches Melo.

- Laboratório de Entomologia, Universidade Federal do Rio de Janeiro, coordenado pelo Prof. Dr. Jorge Luiz Nessimian.

- Laboratório de Entomologia Aquática, Departamento de Biologia da Faculdade de Filosofia Ciências e Letras de Ribeirão Preto, Universidade de São Paulo, coordenado pelo Prof. Dr. Claudio Gilberto Froehlich.

- Laboratório de Entomologia Aquática, Departamento de Hidrobiologia, Universidade Federal de São Carlos, coordenado pela Profa. Dra. Suzana Trivinho Strixino e Profa. Dra. Alaide Aparecida Fonseca Gessner.

- Laboratório de Entomologia Aquática, Departamento de Zoologia, Instituto de Biologia, Universidade Federal da Bahia, coordenado pelo Prof. Dr. Adolfo Ricardo Calor.

- Laboratório de Invertebrados, Museu de História Natural, Pontifícia Universidade Católica de Minas Gerais, coordenado pelo Prof. Dr. Henrique Paprocki.

- Laboratório de Limnologia, Departamento de Biologia Geral, Instituto de Ciências Biológicas, Universidade Federal de Goiás, coordenado pelo Prof. Dr. Leandro Gonçalves de Oliveira.

\section{Principais acervos}

Os acervos brasileiros com o maior número de espécimes de Trichoptera são: 1) Museu de Zoologia da Universidade de São Paulo (MZUSP), onde está depositado o material colecionado dos projetos BIOTA/FAPESP; 2) Museu Nacional, Universidade Federal do Rio de Janeiro (MNRJ); 3) Museu de Zoologia da Universidade Federal da Bahia (UFBA); 4) Acervo do Instituto Nacional de Pesquisas da Amazônia (INPA); 5) Coleção Pe. Jesus Santiago Moure, Universidade Federal do Paraná (DZUP); e 6) Coleção Entomológica Professor José Alfredo Pinheiro Dutra, Departamento de Zoologia, Universidade Federal do Rio de Janeiro (DZRJ). Além destes, material em estudo encontra-se no Laboratório de Entomologia Aquática, Departamento de Biologia, FFCLRP/USP; no Laboratório de Entomologia Aquática, UFBA e no Laboratório de Biologia Aquática, UNESP, campus de Assis, onde também há uma coleção de referência. No exterior, destacam-se os acervos do National Museum of Natural History, Washington, DC, USA (NMNH) e University of Minnesota Insect Collection, Saint Paul, Minnesota, USA (UMSP).

\section{Principais lacunas do conhecimento}

Ainda há grandes áreas do Estado de São Paulo pouco exploradas e outras com estudos incipientes quanto à entomofauna aquática. No caso dos Trichoptera, entre as espécies descritas, o conhecimento dos imaturos é quase insignificante, de maneira que apenas cerca de $2 \%$ das espécies descritas apresentam larvas associadas. Assim, além do esforço para o conhecimento dos adultos de Trichoptera, devem ser estimulados estudos de associação de larvas aos adultos com consequente descrição dos imaturos.

\section{Perspectivas de pesquisa para os próximos 10 anos}

Nos últimos dez anos, o número de pesquisadores que trabalham com Trichoptera aumentou consideravelmente no Brasil, o que foi 
acompanhado pela ampliação do número de publicações tanto em taxonomia, quanto em ecologia deste grupo de insetos. Assim, esperase que, passada mais uma década, o conhecimento da tricopterofauna seja suficiente para implantação de programas de conservação dos recursos hídricos do Estado de São Paulo. Ainda podemos antever o aumento dos estudos filogenéticos com dados morfológicos e moleculares, com resultante busca pela compreensão dos padrões biogeográficos.

\section{Referências Bibliográficas}

ANGRISANO, E.B. \& KOROB, P.G. 2001. Trichoptera. In Guía para la determinación de los artrópodos bentónicos sudamericanos (H.R. Fernández \& E. Domínguez, eds.). Editorial Universitaria de Tucumán, Tucumán, 282p.

CALOR, A.R. 2007. Trichoptera. In Guia on-line: identificação de larvas de insetos aquáticos do Estado de São Paulo. (C.G. Froehlich, org.). Disponível em http://sites.ffclrp.usp.br/aguadoce/guiaonline (último acesso em 18/02/2011).

CALOR, A.R. 2009. Considerações acerca da filogenia de Trichoptera Kirby 1813: da análise dos dados para as hipóteses ou dos cenários para os dados. EntomoBrasilis 2:1-10

CALOR, A.R., AMORIM, D.S. \& HOLZENTHAL, R.W. 2006. Phylogenetics Analysis of Notalina (Neonotalina), with descriptions of two new species. Zootaxa 1131:33-48

CALOR, A.R. \& FROEHLICH, C.G. 2008. Description of immature stages of Notalina morsei Holzenthal, 1986 (Trichoptera: Leptoceridae) and an updated key to Brazilian Leptoceridae genera. Zootaxa 1779:45-54.

CALOR, A.R. \& HOLZENTHAL, R.W. 2008. Phylogeny of Grumichellini Morse, 1981 (Trichoptera: Leptoceridae) with the description of a new genus from southeastern Peru. Aquat. Insect. 30:245-259. http://dx.doi. org/10.1080/01650420802334087

DUMAS, L.L., JARDIM, G.A., SANTOS, A.P.M. \& NESSIMIAN, J.L. 2009. Tricópteros (Insecta: Trichoptera) do Estado do Rio de Janeiro: lista de espécies e novos registros. Arq. Mus. Nac., Rio de Janeiro 67:355-376.

DUMAS, L.L., SANTOS, A.P.M., JARDIM, G.A., FERREIRA-JUNIOR, N. \& NESSIMIAN, J.L. 2010. Insecta, Trichoptera: new records from Brazil and other distributional notes. Check List 6:7-9.

ESKOV, K.Y. \& SUKATCHEVA, I.D. 1997. Geographical distribution of the Paleozoic and Mesozoic caddisflies (Insecta: Trichoptera). In Proceedings of the 8th International Symposium on Trichoptera. (R.W. Holzenthal \& O.S. Flint JUNIOR, eds.). Ohio Biological Survey, Columbus, Ohio, USA, p.95-98.

FLINT JUNIOR, O.S., HOLZENTHAL, R.W. \& HARRIS, S.C. 1999. Catalog of the Neotropical Caddisflies (Insecta: Trichoptera). Ohio Biological Survey, Columbus, 239p.

FRANIA, H.E. \& WIGGINS, G.B. 1997. Analysis of morphological and behavioural evidence for the phylogeny and higher classification of Trichoptera (Insecta). Life Sciences Contribution, Royal Ontario Museum 160:1-67.

FROEHLICH, C.G. 1999. Outros insetos. In Biodiversidade do Estado de São Paulo: síntese do conhecimento ao final do século XX. Invertebrados de Água Doce. (C.A. Joly \& C.E.M. Bicudo, orgs.). FAPESP, São Paulo, v.4, cap.24, p.163-168.

FROEHLICH, C.G. (no prelo). Checklist dos Plecoptera do Estado de São Paulo. Biota Neotrop.

FROEHLICH, C.G., MARIANO, R.L.S., LECCI, L.S \& CALOR, A.R. (dados não publicados). Chave para adultos e estágios imaturos de Ephemeroptera, Plecoptera e Trichoptera do Estado de São Paulo, Brasil. Biota Neotropica.

HENNIG, W. 1969. Die Stammesgeschichte der Insekten. Seckenberg-Büch 49:1-436.

HENNIG, W. 1981. Insect phylogeny (traduzido para o inglês por A.C. Pont \& D. Schlee). Wiley, New York, 439p.
HOLZENTHAL, R.W, BLAHNIK, R.J, KJER, K.M. \& PRATHER, A.P. 2007a. An update on the phylogeny of caddisflies (Trichoptera). In Proceedings of the XII ${ }^{\text {th }}$ International Symposium on Trichoptera (J. Bueno-Soria, R. Barba-Alvarez \& B. Armitage, eds.). The Caddis Press, Columbus, Ohio, p.13-153.

HOLZENTHAL, R.W, BLAHNIK, R.J, PRATHER, A.P \& KJER, K.M 2007b. Order Trichoptera Kirby, 1813 (Insecta), Caddisflies. Zootaxa 1668:639-698.

IVANOV, V.D. 1997. Rhyacophiloidea: a paraphyletic taxon. In Proceedings of the 8th International Symposium on Trichoptera ( R.W. Hozenthal \& O.S. Flint JUNIOR, eds.). Ohio Biological Survey, Columbus, Ohio, USA, 496p.

KJER, K.M. 2004. Aligned 18S and insect phylogeny. Syst. Biol. 53:506-514.

KJER, K.M., BLAHNIK, R.J. \& HOLZENTHAL, R.W. 2001. Phylogeny of Trichoptera (Caddisflies): characterization of signal and noise within multiple datasets. Syst. Biol. 50(6):781-816. http://dx.doi. org/10.1080/106351501753462812

KJER, K.M., BLAHNIK, R.J. \& HOLZENTHAL, R.W. 2002. Phylogeny of caddisflies (Insecta, Trichoptera). Zool. Scr. 31:83-91.

KRISTENSEN, N.P. 1984. Studies on the morphology and systematics of primitive Lepidoptera (Insecta). Steenstrupia 10:141-191.

KRISTENSEN, N.P. 1991. Phylogeny of extant hexapods. In The Insects of Australia. Commonwealth Scientific and Industrial Research Organization, ed. Cornell University Press, Ithaca, 125-140

MORETTO, R.A., CALOR, A.R. \& FROEHLICH, C.G. 2008. Levantamento de Trichoptera Kirby, 1813 do Parque Nacional Serra do Divisor, Acre: 1. Hydropsychidae. In Livro de resumos do XXII Congresso Brasileiro de Entomologia, Uberlândia, Minas Gerais.

MORSE, J.C. 1997. Phylogeny of Trichoptera. Annu. Rev. Entomol. 42:42750.

MORSE, J.C. 2010. Trichoptera world checklist. Disponível em: http://entweb. clemson.edu/database/trichopt/index.htm (último acesso em 16/08/2010).

NEBOISS, A. 1991. Trichoptera. In The insects of Australia: a textbook for students and researchs workers. 2nd ed. (I.D. Nauman, P.B. Carne, J.F. Laurence, E.S. Nielsen \& J.P. Spradbury, eds.). Cornell Univ. Press, Ithaca, NY, v.2, p.787-816.

PAPROCKI, H., HOLZENTHAL, R.W. \& BLAHNIK, R.J. 2004. Checklis of the Trichoptera (Insecta) of Brazil I. Biota Neotrop.: http://www. biotaneotropica.org.br/v4n1/pt/fullpaper?bn01204012004+en (último acesso em 18/02/2011).

PES, A.M.O., HAMADA, N. \& NESSIMIAN, J.L. 2005. Chave de identificação de larvas para famílias e gêneros de Trichoptera (Insecta) da Amazônia Central, Brasil. Rev. Bras. Entomol. 49:181-204.

QUINTEIRO, F.B., CALOR, A.R. \& FROEHLICH, C.G. 2011. A new species of Phylloicus Müller 1880 (Trichoptera: Calamoceratidae) from Southeastern Brazil, including description of larval and pupal stages. Zootaxa 2748: 38-46.

ROSS, H.H. 1967. The evolution and the past dispersal of the Trichoptera. Annu. Rev. Entomol. 12:169-206.

SPIES, M.R. \& FROEHLICH, C.G. 2009. Inventory of caddisflies (Trichoptera: Insecta) of the Campos do Jordão State Park, São Paulo state, Brazil. Biota Neotrop.: http://www.biotaneotropica.org.br/v9n4/pt/ fullpaper?bn03509042009+en (último acesso em 18/02/2011).

TILLYARD, R.J. 1935. The evolution of the scorpion-flies and their derivatives (Order Mecoptera). Ann. Entomol. Soc. Am. 28:1-45.

WEAVER III, J.S. 1984. The evolution and classification of Trichoptera, Part I: the Groundplan of Trichoptera. In Proceedings of the $4^{\text {th }}$ International Symposium on Trichoptera (J.C. Morse, ed.). Junk, Series Entomologica, p.413-419.

WHEELER, W.C., WHITING, M.F., WHEELER, Q.D. \& CARPENTER, J.M. 2001. The phylogeny of extant insect orders. Cladistics 17:113-169.

WIGGINS, G.B. \& WICHARD, W. 1989. Phylogeny of pupation in Trichoptera, with proposals on the origin and higher classification of the order. J. N. Am. Benthol. Soc. 8:260-276. 


\section{Apêndices}

Apêndice 1. Lista de teses e dissertações que incluíram espécies da ordem Trichoptera no Estado de São Paulo.

Appendix 1. $\mathrm{PhD}$ thesis and dissertations that included Trichoptera species in São Paulo State.

\section{Teses}

1996 Leandro Gonçalves Oliveira: “Aspectos da biologia de comunidades de insetos aquáticos da ordem Trichoptera Kirby, 1813, em córregos de cerrado do município de Pirenópolis, Estado de Goiás”. PPG Entomologia, FFCLRP-USP. Orientador: Dr. Claudio G. Froehlich.

2002 Adriano Sanches Melo: "Estudos sobre estimadores de riqueza de espécies, perturbações experimentais e persistência ao longo de cinco anos em comunidades de macroinvertebrados em riachos". PPG Ecologia, UNICAMP. Orientador: Dr. Claudio G. Froehlich.

2002 Pitágoras da Conceição Bispo: "Estudo de comunidades de Ephemeroptera, Plecoptera e Trichoptera (EPT) em riachos do Parque Estadual Intervales, Serra de Paranapiacaba, Sul do Estado de São Paulo”. PPG Zoologia, IBUSP. Orientador: Dr. Claudio G. Froehlich.

2003 Vera Lúcia Crisci-Bispo: "Ecologia de imaturos de Ephemeroptera, Plecoptera e Trichoptera (EPT) associados ao substrato rochoso e ao folhiço, e dinâmica de colonização de macroinvertebrados aquáticos em riachos do Parque Estadual Intervales". PPG Biologia Comparada, FFCLRP-USP. Orientador: Dr. Claudio G. Froehlich.

2006 Juliano José Corbi: "Influência de diferentes usos da terra sobre os macroinvertebrados aquáticos de córregos: ênfase para o cultivo de cana-de-açúcar em áreas adjacentes". PPG Ecologia e Recursos Naturais, UFSCar. Orientadora: Dra. Susana Trivinho-Strixino.

2008 Adolfo Ricardo Calor: "Filogenia de Grumichellini Morse, 1981 (Trichoptera: Leptoceridae: Triplectidinae) e revisão taxonômica de Grumichella Müller, 1879”. PPG Entomologia, FFCLRP-USP. Orientador: Dr. Claudio G. Froehlich.

2008 Marcia Thais Suriano: "Macroinvertebrados bentônicos em córregos de baixa ordem sob diferentes usos do solo no Estado de São Paulo: subsídios para biomonitoramento". PPG Ecologia e Recursos Naturais, UFSCar. Orientadora: Dra. Alaíde Aparecida Fonseca Gessner.

2009 Marcia Regina Spies: "Estrutura das comunidades de larvas de Trichoptera Kirby, 1813 (Insecta) em riachos do Parque Estadual de Campos do Jordão, São Paulo, Brasil”. PPG Entomologia, FFCLRP-USP. Orientador: Dr. Claudio G. Froehlich.

\section{Dissertações}

1991 Leandro Gonçalves Oliveira: "Estudo da fauna de Trichoptera do córrego do Pedregulho, Pedregulho, SP, com especial referência à família Hydropsychidae". PPG Entomologia, FFCLRP-USP. Orientador: Dr. Claudio G. Froehlich.

1998 Adriano Sanches Melo: "Macroinvertebrados associados a pedras em riachos: padrões de diversidade ao longo de uma bacia hidrográfica”. PPG Ecologia, UNICAMP. Orientador: Dr. Claudio G. Froehlich.

1998 Pitágoras da Conceição Bispo: "Estudo da Fauna de Plecoptera em Córregos de uma região Serrana do Brasil Central”. PPG Zoologia, IBUSP. Orientador: Dr. Claudio G. Froehlich.

1997 Henrique Paprocki: “Aspectos da Ecologia de Insetos Aquáticos em dois Riachos de Primeira Ordem da Serra do Cipó/MG”. PPG Entomologia, FFCLRP-USP. Orientador: Dr. Claudio G. Froehlich.

2004 Adolfo Ricardo Calor: "Sistemática filogenética e aspectos biogeográficos de Hudsonemini (Trichoptera: Leptoceridae)". PPG Entomologia, FFCLRP-USP. Orientador: Dr. Dalton S. Amorim.

2007 Priscilla Kleine: "Macroinvertebrados em córregos da região da Mata Atlântica (Sudeste do Brasil): Influência do cultivo de banana". PPG Ecologia e Recursos Naturais, UFSCar. Orientadora: Dra. Susana Trivinho-Strixino.

2008 Márcia Cristina de Paula: "Macroinvertebrados em córregos em fragmentos de mata com diferentes estados de conservação no interior do Estado de São Paulo". PPG Ecologia e Recursos Naturais, UFSCar. Orientadora: Dra. Alaíde Aparecida Fonseca Gessner.

2008 Elisa Yokoyama: "Distribuição espacial de Trichoptera (Insecta) em riachos de montanha do Sul do Estado de São Paulo". PPG Entomologia, FFCLRP-USP. Orientador: Dr. Pitágoras da Conceição Bispo.

Apêndice 2. Lista bibliográfica de trabalhos que incluem Trichoptera do Estado de São Paulo.

Appendix 2. List of papers that included Trichoptera species from São Paulo State.

ALMEIDA, G.L. \& FLINT JUNIOR, O.S. 2002. Five new species of Smicridea McLachlan (Trichoptera: Hydropsychidae) from Brazil. Rev. Bras. Zool. 19(3):767-775

BANKS, N. 1915 [1914]. New neuropteroid insects, native and exotic. P. Acad. Nat. Sci. Phila. 66:608-632.

BLAHNIK, R.J. 1997. Systematics of Chimarrita, a new subgenus of Chimarra (Trichoptera: Philopotamidae). Syst. Entomol. 22:199-243.

BLAHNIK, R.J. 1998. A revision of the Neotropical species of the genus Chimarra, subgenus Chimarra (Trichoptera: Philopotamidae). Mem. Am. Entomol. Inst. 59:1-318.

BLAHNIK, R.J. 2002. Systematics of Otarrha, a new Neotropical subgenus of Chimarra (Trichoptera: Philopotamidae). Syst. Entomol. 27:65-130.

BLAHNIK, R.J. 2005. Alterosa, a new caddisfly genus from Brazil (Trichoptera: Philopotamidae). Zootaxa 991:1-60.

BLAHNIK, R.J., PAPROCKI, H. \& HOLZENTHAL, R.W. 2004. New distribution and species records of Trichoptera from Southern and Southeastern BraziL. Biota Neotrop.: http://www.biotaneotropica.org.br/v4n1/pt/fullpaper?bn01304012004+en (último acesso em 21/02/2011).

BRAUER, F. 1865. Zweiter berichte über die auf der Weltfahrt der Kais. Fregatte Novara gesammelten Neuropteren. Verhandlungen der Kaiserlich-königlichen Zoologischen-Botanischen Gesellschaft in Wien 15:415-422.

BURMEISTER, H.C.C. 1839. Handbuch der entomologie. Zweiter Band, Zweite Abtheilung, Theod. Chr. Friedr. Enslin, Berlin, xii + 397-1050p.

CALOR, A.R. 2007. Trichoptera. In Guia on-line: identificação de larvas de Insetos aquáticos do Estado de São Paulo (C.G. Froehlich, org.). Disponível em: http://sites.ffclrp.usp.br/aguadoce/guiaonline (último acesso em 18/02/2011). 
CALOR, A.R., AMORIM, D.S. \& HOLZENTHAL, R.W. 2006. Phylogenetics Analysis of Notalina (Neonotalina), with descriptions of two new species. Zootaxa 1131:33-48

CALOR, A.R. \& FROEHLICH, C.G. 2008. Description of immature stages of Notalina morsei Holzenthal, 1986 (Trichoptera: Leptoceridae) and an updated key to Brazilian Leptoceridae genera. Zootaxa 1779:45-54. http://dx.doi.org/10.1080/01650420802334087

CALOR, A.R. \& HOLZENTHAL, R.W. 2008. Phylogeny of Grumichellini Morse, 1981 (Trichoptera: Leptoceridae) with the description of a new genus from southeastern Peru. Aquat. Insect. 30:245-259.

CHAMORRO, M.L. \& HOLZENTHAL, R.W. 2010. Taxonomy and phylogeny of New World Polyplectropus Ulmer, 1905 (Trichoptera: Psychomyioidea: Polycentropodidae) with the description of 39 Chamorro \& Holzenthal, 2010. Zootaxa 2582:1-252.

CRISCI-BISPO, V.L.,BISPO, P.C. \& FROEHLICH, C.G. 2004. Triplectides in empty cases of Nectopsyche (Trichoptera: Leptoceridae), Intervales State Park, São Paulo State, Brazil. Nota Científica. Rev. Bras. Entomol. 48(1):133-134.

CRISCI-BISPO, V.L., BISPO, P.C. \& FROEHLICH, C.G. 2007a. Ephemeroptera, Plecoptera and Trichoptera assemblages in two Atlantic Rainforest streams, Southeastern Brazil. Ver. Bras. Zool. 24(2):312-318. http://dx.doi.org/10.1590/S0101-81752007000200007

CRISCI-BISPO, V.L., BISPO, P.C. \& FROEHLICH, C.G. 2007b. Ephemeroptera, Plecoptera and Trichoptera assemblages in litter in a mountain stream of the Atlantic Rainforest from Southeastern Brazil. Rev. Bras. Zool. 24(3):545-551.

DENNING, D.G. \& SIKORA, J. 1968. Three new species of Trichoptera from Brazil. Beiträge zur Neotropischen Fauna 5: $72-177$.

DUMAS, L.L. \& NESSIMIAN, J.L. 2008. A new species of Neoatriplectides Holzenthal, 1997 (Insecta: Trichoptera: Atriplectididae), from Brazil, including description of the pupa of the genus. Zootaxa 1773:63-68.

DUMAS, L.L., SANTOS, A.P.M., JARDIM, G.A., FERREIRA-Jr, N. \& NESSIMIAN, J.L. 2010. Insecta, Trichoptera: New records from Brazil and other distributional notes. Check List 6:7-9.

FLINT JUNIOR, O.S. 1967. Studies of Neotropical caddis flies, V: types of the species described by Banks and Hagen. Proceedings of the United States National Museum 123(3619):1-37.

FLINT JUNIOR, O.S. 1972. Studies of Neotropical caddis flies, XIV: on a collection from northern Argentina. P. Biol. Soc. Wash. 85:223-248.

FLINT JUNIOR, O.S. 1973. Studies of Neotropical caddis flies, XVI: the genus Austrotinodes (Trichoptera: Psychomyiidae). P. Biol. Soc. Wash. 86:127-142.

FLINT JUNIOR, O.S. 1974. Studies of Neotropical caddisflies, XVII: the genus Smicridea from North and Central America (Trichoptera: Hydropsychidae). Sm. C. Zool. 167:1-65.

FLINT JUNIOR, O.S. 1978. Studies of Neotropical caddisflies, XXII: Hydropsychidae of the Amazon basin (Trichoptera). Amazoniana 6:373-421.

FLINT JUNIOR, O.S. 1980. Studies of Neotropical caddisflies, XXVI: new species from Argentina (Trichoptera). Revista de La Sociedad Entomologica Argentina 39:137-142.

FLINT JUNIOR, O.S. 1983. Studies of Neotropical caddisflies, XXXIII: new species from austral South America (Trichoptera). Sm. C. Zool. 377:1-100.

FLINT JUNIOR, O.S. 1996. Trichoptera collected on the expeditions to Parque Manu, Madre de Dios, Peru. In Manu: the biodiversity of southeastern Peru (D.E. Wilson \& A. Sandoval, eds.). Smithsonian Institution Press, Washington, DC, p.369-430.

FLINT JUNIOR, O.S.1998. Studies of Neotropical caddisflies, LIII: a taxonomic revision of the subgenus Curgia of the genus Chimarra (Trichoptera: Philopotamidae). Sm. C. Zool. 594:1-131.

FLINT JUNIOR, O.S. \& DENNING, D.G. 1989. Studies of Neotropical caddisflies, XLI: new species and records of Austrotinodes (Trichoptera: Psychomyiidae). Pan-Pacific Entomologist 65:108-122.

FLINT JUNIOR, O.S., HOLZENTHAL, R.W. \& HARRIS, S.C. 1999. Catalog of the Neotropical Caddisflies (Insecta: Trichoptera). Ohio Biological Survey, Columbus, 239p.

FLINT JUNIOR, O.S., McALPINE, J.F. \& ROSS, H.H. 1987. A revision of the genus Leptonema Guérin (Trichoptera: Hydropsychidae). Sm. C. Zool. 450:1-193. FROEHLICH, C.G. 1999. Outros insetos. In Biodiversidade do Estado de São Paulo: síntese do conhecimento ao final do século XX. Invertebrados de Água Doce ( C.A Joly \& C.E.M. Bicudo, orgs.). FAPESP, São Paulo, v.4, cap.24.

FROEHLICH, C.G. 2001. Insetos Aquáticos. In Intervales. Fundação para a Conservação e a Produção Florestal do Estado de São Paulo. Governo do Estado de São Paulo; Secretaria de Estado do Meio Ambiente, São Paulo, p.181-188.

GUÉRIN-MENEVILLE, F.E. 1843. Insectes. In Iconographie du règne animal du Cuvier. J.B. Baillière, Paris, 576p + 104 pls.

HARRIS, S.C., FLINT JUNIOR, O.S. \& HOLZENTHAL, R.W. 2002. Review of the Neotropical genus Flintiella (Trichoptera: Hydroptilidae: Stactobiini). J. New York Entomol. S. 110(1):65-90.

HOLZENTHAL, R.W. 1984. Studies in Neotropical Leptoceridae (Trichoptera) I: Achoropsyche, a new genus. In Proceedings of the $4^{\text {th }}$ International Symposium on Trichoptera (J.C. Morse, ed.). Junk, The Hague, 181-184.

HOLZENTHAL, R.W. 1986. The Neotropical species of Notalina, a southern group of long-horned caddisflies (Trichoptera: Leptoceridae). Syst. Entomol. 11:61-73.

HOLZENTHAL, R.W. 1988a. Studies in Neotropical Leptoceridae (Trichoptera), VIII: the genera Atanatolica Mosely and Grumichella Müller (Triplectidinae: Grumichellini). T. Am. Entomol. Soc. 114:71-128.

HOLZENTHAL, R.W. 1988b. Systematics of Neotropical Triplectides (Trichoptera: Leptoceridae). Ann. Entomol. Soc. Am. 81:187-208.

HOLZENTHAL, R.W. 1989. Studies in Neotropical Leptoceridae (Trichoptera), IX: a new genus and species from southeastern Brazil. Aquatic Insects 11:29-32.

HOLZENTHAL, R.W. 1995. The Caddisfly genus Nectopsyche: New gemma group species from Costa Rica and the Neotropics Trichoptera: Leptoceridae). J. N. Am. Benthol. Soc. 14:61-83.

HOLZENTHAL, R.W. 1997. The caddisfly (Trichoptera) family Atriplectididae in the Neotropics. In Proceedings of the $8^{\text {th }}$ International Symposium on Trichoptera (R.W. Holzenthal \& O.S. Flint Junior, eds.). Ohio Biological Survey, Columbus, p.157-165.

HOLZENTHAL, R.W. \& ROBERTSON, D.R. 2006. Four new species of Contulma from South America (Trichoptera: Anomalopsychidae). Zootaxa 1355:49-59. KOLENATI, F.A. 1859. Genera et Species Trichopterorum, Pars Altera. Nouveaux Mémoires de la Société Impériale des Naturalistes de Moscou 11:141-296. MARLIER, G. 1964. Sur trois Trichopteres nouveaux recueillis en Amerique du Sud par le Professeur J. Illies. Bull. Institut Roy. Sci. Natur. Belg. 40(6):1-15. 
MELO, A.S. \& FROEHLICH, C.G. 2001. Macroinvertebrates in neotropical streams: richness patterns along a catchment and assemblage structure between 2 seasons. J. N. Am. Benthol. Soc. 20(1):1-16.

MELO, A.S. \& FROEHLICH, C.G. 2004. Colonization by macroinvertebrates of experimentally disturbed stones in three tropical streams differing in size. Int. Rev. Hydrobiol. 89(3):317-325.

MOSELY, M.E. 1934. Some new exotic Trichoptera. Stylops 3:139-142.

MOSELY, M.E. 1936. A revision of the Triplectidinae, a subfamily of the Leptoceridae (Trichoptera). T. Roy. Ent. Soc. London 85:91-130.

MÜLLER, F. 1879. Notes on the cases of some south Brazilian Trichoptera. T. Roy. Ent. Soc. London 1879:131-144.

MÜLLER, F. 1880a [1878]. Sobre as casas construidas pelas larvas de insectos Trichopteros da Provincia de Santa Catharina. Archivos do Museu Nacional 3:99-134, 210-214, pranchas 8-11.

MÜLLER, F. 1880b. Über die von den Trichopterenlarven der Provinz Santa Catharina verfertigen Gehäuse. Z. Wiss. Zool. 35:47-87.

NAVÁS, R.P.L. 1916. Neurópteros Sudamericanos. Tercera Serie. Brotéria, Série Zoológica 14:14-35.

OLIVEIRA, L.G. \& FROEHLICH, C.G. 1996. Natural history of three Hydropsychidae (Trichoptera, Insecta) in a "Cerrado" stream from northeastern São Paulo, Brazil. Ver. Bras. Zool. 13:755-762.

PAPROCKI, H., HOLZENTHAL, R.W. \& BLAHNIK, R.J. 2004. Checklist of the Trichoptera (Insecta) of Brazil I. Biota Neotrop.: http://www.biotaneotropica. org.br/v4n1/pt/fullpaper?bn01204012004+en (último acesso em 18/02/2011).

PERTY, J.A.M. 1833. Insecta Brasiliensia. In Delectus animalium articulatorum (K.F.P. Martius, ed.). Impensis Editoris, Münich, p.1-224.

PRATHER, A.L. 2003. Revision of the Neotropical caddisfly genus Phylloicus (Trichoptera: Calamoceratidae). Zootaxa 275:1-214.

ROBERTSON, D.R. \& HOLZENTHAL, R.W. 2005. Revision of the genus Culoptila (Trichoptera: Glossosomatidae). Zootaxa 1233:1-52.

ROSS, H.H. 1947. Descriptions and records of North American Trichoptera, with synoptic notes. T. Am. Entomol. Soc. 73:125-168.

SCHMID, F. 1982. La famille des Xiphocentronides (Trichoptera: Annulipalpia). Mém. Soc. Entomol. Canada 121:1-127.

SCHMID, F. 1989. Les Hydrobiosides (Trichoptera, Annulipalpia). Bull. Institut Roy. Sci. Natur. Belg. 59(Suppl.):1-154.

SPIES, M.R. \& FROEHLICH, C.G. 2009. Inventory of caddisflies (Trichoptera: Insecta) of the Campos do Jordão State Park, São Paulo state, Brazil. Biota Neotrop.: http://www.biotaneotropica.org.br/v9n4/pt/fullpaper?bn03509042009+en (último acesso em 18/02/2011). http://dx.doi.org/10.1590/S167606032009000400021

THOMSON, R.E. \& HOLZENTHAL, R.W. 2010. New Neotropical species of the genus Austrotinodes Schmid (Trichoptera: Ecnomidae). Zootaxa 2437:38-50.

ULMER, G. 1905. Zur Kenntniss aussereuropäischer Trichopteren. Stettiner Entomologische Zeitung 66:1-119.

ULMER, G. 1907. Neue Trichopteren. Notes from the Leyden Museum 29:1-53.

ULMER, G. 1911. Einige Südamerikanische Trichopteren. Ann. Soc. Entomol. Belg. 55:15-26.

ULMER, G. 1913. Verzeichnis der südamerikanischen Trichopteren, mit Bemerkungen über einzelne Arten. Deut. Entomol. Z. 1913:383-414.

ULMER, G. 1955. Köcherfliegen (Trichopteren) von den Sunda- Inseln (Teil II). Arch. Hydrobiol. 21 (Suppl.):408-608.

VALLOT, J.J. 1855 [1854]. Sur deux fourreaux hélicoïdes façonnés par les larves d'insectes. Mémoires de l'Académie dês Sciences, Arts et Belles-lettres de Dijon 3:10-12.

WASMUND, A.M. \& HOLZENTHAL, R.W. 2007. A revision of the Neotropical caddisfly genus Rhyacopsyche, with the description of 13 new speciesT (Trichoptera: Hydroptilidae). Zootaxa 1634:1-59. 